\title{
MHD transient free convection and chemically reactive flow past a porous vertical plate with radiation and temperature gradient dependent heat source in slip flow regime
}

\author{
B. Madhusudhana Rao ${ }^{*}$ G.Viswanatha Reddy ${ }^{* * *}$ M.C.Raju ${ }^{* *}$ S.V.K.Varma*** \\ *Department of Mathematics, R.M.K. Engineering College, Chennai. \\ ** Department of H\&S, Annamacharya Institute of Technology and Sciences (Autonomous), Rajampet. \\ *** Department of Mathematics,S.V.University,Tirupati - 517502
}

\begin{abstract}
The present paper deals with the analysis of unsteady free convection heat and mass transfer flow through a non-homogeneous porous medium with variable permeability bounded by an infinite porous vertical plate in slip flow regime taking into account the radiation, chemical reaction and temperature gradient dependent heat source. The flow is considered under the influence of magnetic field applied normal to the flow. The permeability of the porous medium and the suction velocity at the plate decrease exponentially with time about a constant mean. Approximate solutions for velocity, temperature and concentration fields are obtained using perturbation technique. The expressions for skin-friction and rate of heat transfer and rate of mass transfer are also derived. The results obtained are discussed for cooling case $(\mathrm{Gr}>0)$ of the plate. The effects of various physical parameters, encountered into the problem, on the velocity field are numerically shown through graphs while the effects on skin-friction and rate of heat and mass transfer are numerically discussed with the help of tables.
\end{abstract}

Key words: MHD, Free convection, heat and mass transfer, radiation, non homogeneous porous medium, temperature gradient dependant heat source and chemical reaction.

\section{Introduction}

Free convection arises in the fluids when temperature changes cause density variation leading to buoyancy forces acting on the fluid elements. The most common example of free convection is the atmospheric flow which is driven by temperature differences. Sometimes along with the free convection currents caused by difference in temperature the flow is also affected by the differences in concentration or material constitution. This type of flow has applications in many branches of science and engineering. The study of such flow under the influence of magnetic field has attracted the interest of many investigators in view of its application in MHD generators, plasma studies, nuclear reactors, geothermal.Hydro magnetic combined heat and mass transfer by natural convection from a permeable surface embedded in a fluid saturated porous medium was studied by Chamkha [1-3].

The fluid under consideration there does occur some chemical reaction e.g. air and benzene react chemically, so also water and sulfuric acid. During such chemical reactions, there is always generation of heat. Combining heat and mass transfer problems with a chemical reaction have importance in many processes and have therefore received a considerable amount of attention in recent years. In many chemical engineering processes chemical reactions take place between a foreign mass and working fluid which moves due to the stretch of a surface. The order of the chemical reactions depends on several factors. One of the simplest chemical reactions is the first-order reaction in which the rate of the reaction is directly proportional to the species concentration. The chemical reactions can be codified as either heterogeneous or homogenous processes. In most cases of chemical reactions the reaction rate depends on the concentration of the species itself. If the rate of reaction is directly proportional to the concentration then the reaction is said to homogeneous reaction or first order reaction. The analysis of the nonlinear MHD flow with heat and mass transfer characteristics of an incompressible, viscous, electrically conducting, and Boussinesq fluid on a vertical stretching surface with a chemical reaction and thermal stratification effects was obtained by Kandasamy et al. [1]. The heat and mass transfer characteristics of the natural convection about a vertical surface embedded in a saturated porous medium subjected to a chemical reaction taking into account the Soret and Dufour effect was analyzed by Postelnicu [2]. Ibrahim etal. [3] investigated the effect of the chemical reaction and radiation absorption on the unsteady MHD free convection flow past a semi-infinite vertical permeable moving plate with the heat source and suction.

In many practical applications, the particle adjacent to a solid surface no longer takes the velocity of the surface. The particle at the surface has a finite tangential velocity; it "slips" along the surface. The flow regime is called the slip -flow regime and this effect cannot be neglected. Using these assumptions, Sharma and Chaudhary 
[5], discussed the free convection flow past a vertical plate in slip-flow regime and also discussed the free convection flow past a vertical plate in slip-flow regime and also discussed its various applications for engineering purpose. Also, Sharma [6], investigate the effect of periodic heat and mass transfer on the unsteady free past a vertical flat plate convection flow in slip-flow regime when suction velocity oscillates in time. Coupled non-linear partial differential equations governing free convection flow, heat and mass transfer has been obtained analytically using the perturbation technique. The fluids considered in this investigation are air $(\operatorname{Pr}=0.71)$ and water $(\mathrm{Pr}=7)$ in the presence of Hydrogen $(\mathrm{Sc}=0.22)$. Magneto hydrodynamic convective heat and mass transfer in a boundary layer slip flow past a vertical permeable plate with thermal radiation and chemical reaction were investigated by Pal and Talukda [4].Kumar et al. [11] studied the fluctuating flow of elastic-viscous stratified fluid through a porous medium past an infinite rigid plane in slip flow regime in the presence of transverse magnetic field. Gupta and Sharma [12] , Sing et al. [13-15], have studied MHD flow through porous medium in slip regime. Recently, Jaiswal and Soundalgekar [16] have discussed the flow past an infinite vertical plate oscillating in its plane in the presence of a temperature gradient dependent heat source. while Taneja and Jain [17] have presented a theoretical analysis for unsteady free convection flow with radiation in slip flow regime.Yamamoto and yoshida [7] studied flow with convective acceleration through a porous medium considering suctionand injection flow through a plane porous wall and generalization of this study has been presented by Yamamoto and Iwamura [8]. Daniels et al.[9-10] have discussed thermally driven shallow cavity flows in porous medium for the intermediate regime and merged layer regime respectively.

The object of the present paper is to study the unsteady MHD free convection flow of a viscous fluid past a vertical porous plate embedded with porous medium in presence of chemical reaction.In obtaining the solution, the terms regarding radiation effect, temperature gradient dependent heat source are taken into account of energy equation and chemical reaction parameter is taken into account of concentration equation. The Permeability of the porous medium and the suction velocity are consideredto be as exponentially decreasing function of time.

\section{Mathematical Analysis:}

We consider a two-dimensional unsteady free convection flow of an incompressible viscous fluid past an infinite vertical porous plate. In rectangular Cartesian coordinate system, we take x-axis along the plate in the direction of flow and y-axis normal to it. Further the flow is considered in presence of temperature gradient dependent heat source and effect of radiation and chemical reaction.

In the analysis the magnetic Reynolds number is taken to be small so that the induced magnetic field is neglected. Likewise for small velocity the viscous dissipation and Darcy's dissipation are neglected. The flow in the medium is entirely due to buoyancy force caused by temperature difference between the porous plate and the fluid. Under the above assumptions, the equations governing the conservation of mass (continuity), momentum, energy and concentration can be written as follows:

$$
\begin{aligned}
& \frac{\partial v^{1}}{\partial y^{1}}=0 \\
& \frac{\partial u^{1}}{\partial t^{1}}+v^{1} \frac{\partial u^{1}}{\partial y^{1}}=g \beta_{1}\left(T^{1}-T_{\infty}^{1}\right)+g \beta_{2}\left(C^{1}-C_{\infty}^{1}\right)+v \frac{\partial^{2} u^{1}}{\partial y^{1^{2}}}-\frac{v}{k^{1}(t)} u^{1}-\frac{\sigma B_{0}{ }^{2}}{\rho} u \\
& \frac{\partial T^{1}}{\partial t^{1}}+v^{1} \frac{\partial T^{1}}{\partial y^{1}}=\frac{K_{T}}{\rho C_{p}} \frac{\partial^{2} T^{1}}{\partial y^{1^{2}}}-\frac{1}{\rho C_{p}} \frac{\partial q_{r}}{\partial y^{1}}+\frac{Q^{1}}{\rho C_{p}} \frac{\partial T}{\partial y} \\
& \frac{\partial C^{1}}{\partial t^{1}}+v^{1} \frac{\partial C^{1}}{\partial y^{1}}=D \frac{\partial^{2} C^{1}}{\partial y^{1^{2}}}-K_{c}{ }^{1}\left(C^{1}-C_{\infty}^{1}\right)
\end{aligned}
$$

The boundary conditions relevant to the problem are;

$$
\begin{array}{lrlrl}
u^{1}=L_{1}\left(\frac{\partial u^{1}}{\partial y^{1}}\right), & T^{1}=T_{w}^{1}, & C^{1}=C_{w}^{1} & \text { at } \quad y^{1}=0 \\
u^{1} \rightarrow 0, & T^{1} \rightarrow T_{\infty}^{1}, & C^{1} \rightarrow C_{\infty}^{1} & \text { as } y^{1} \rightarrow \infty
\end{array}
$$


Where $u^{1}$ and $v^{1}$ are the components of velocity along $\mathrm{x}$-axis and y-axis directions, $t$ is the time, $g$ is the acceleration due to gravity, $\beta_{1}$ and $\beta_{2}$ are the coefficients of volume expansion, $v$ is the kinematic viscosity, $k^{1}(t)$ is the permeability of the porous medium, $\rho$ is the density of the fluid, $\sigma$ is the electrical conductivity of the fluid, $B_{0}$ is the uniform magnetic field, $T^{1}$ is the temperature, $K_{T}$ is the thermal conductivity, $C_{p}$ is the specific heat at constant pressure, $q_{r}$ is the radioactive heat flux, $Q^{1}$ is the heat source, $T_{w}^{1}$ is the temperature of the wall as well as the temperature of the fluid at the plate, $T_{\infty}^{1}$ is the temperature of the fluid far away from the plate, $L_{1}=\left(\frac{2-m_{1}}{m_{1}}\right)$ being the mean free path where $m_{1}$ is the Maxwell's reflection coefficient, $C^{1}$ is the concentration, $D$ is the density, $K_{c}{ }^{1}$ is chemical reaction parameter and $C_{\infty}^{1}$ is the concentration of the wall as well as the concentration of the fluid at the plate.

The equation of continuity (1) yields that $\mathrm{v}^{1}$ is either a constant or some function of time, hence we assume that

$$
v^{1}=-v_{0}^{1}\left(1+\varepsilon e^{-n^{1} t^{1}}\right)
$$

Where $v_{0}^{1}>0$ is the suction velocity at the plate and $n^{1}$ is a positive constant. The negative sign indicates that the suction velocity acts towards the plate.

Consider the fluid which is optically thin with a relatively low density and radioactive heat flux is given by Ede [18] in the following;

$$
\frac{\partial q_{r}}{\partial y^{1}}=4\left(T^{1}-T_{\infty}^{1}\right) I
$$

Where $I$ is the absorption coefficient at the plate.

The Permeability $k^{1}(t)$ of the porous medium is considered in the following form

$$
k^{1}(t)=k_{0}^{1}\left(1+\varepsilon e^{-n^{1} t^{1}}\right)
$$

On introducing the following dimensionless quantities and variable

$$
\begin{aligned}
& u=\frac{u^{1}}{v_{0}^{1}}, \quad y=\frac{y^{1} v_{0}^{1}}{v}, \quad n=\frac{4 v n^{1}}{v_{0}^{1^{2}}}, G_{r}=\frac{g \beta v\left(T_{w}^{1}-T_{\infty}^{1}\right)}{v_{0}^{1^{3}}}, t=\frac{v_{0}^{1^{2}} t^{1}}{4 v}, M=\frac{\sigma B_{0}{ }^{2} v}{v_{0}^{1^{3}}}, \\
& T=\frac{T^{1}-T_{\infty}^{1}}{T_{w}^{1}-T_{\infty}^{1}}, K_{0}=\frac{K_{0}^{1} v_{0}^{2}}{v^{2}}, R=\frac{4 v I}{\rho C_{p} v_{0}^{1^{2}}}, S_{c}=\frac{v}{D}, H=\frac{Q^{1} v}{\rho C_{p} v_{0}^{1^{2}}\left(T_{w}^{1}-T_{\infty}^{1}\right)}, P_{r}=\frac{\rho v C_{p}}{K T}, \\
& C=\frac{C^{1}-C_{\infty}^{1}}{C_{w}^{1}-C_{\infty}^{1}}, K_{c}=\frac{K_{c}^{1} v}{v_{0}^{1^{2}}} .
\end{aligned}
$$

in set of equations (2)-(4), we obtain the non-dimensional form of the governing equations as follows:

$$
\begin{aligned}
& \frac{1}{4} \frac{\partial u}{\partial t}-\left(1+\varepsilon e^{-n t}\right) \frac{\partial u}{\partial y}=G_{r} T+G_{m} C+\frac{\partial^{2} u}{\partial y^{2}}-\left[M+\frac{1}{k_{0}\left(1+\varepsilon e^{-n t}\right)}\right] u \\
& \frac{1}{4} \frac{\partial T}{\partial t}-\left(1+\varepsilon e^{-n t}\right) \frac{\partial T}{\partial y}=\frac{1}{P_{r}} \frac{\partial^{2} T}{\partial y^{2}}-R T+H \frac{\partial T}{\partial y} \\
& \frac{1}{4} \frac{\partial C}{\partial t}-\left(1+\varepsilon e^{-n t}\right) \frac{\partial C}{\partial y}=\frac{1}{S_{c}} \frac{\partial^{2} C}{\partial y^{2}}-k_{c} C
\end{aligned}
$$

And boundary conditions (5) reduce to

$$
\begin{aligned}
& u=h\left(\frac{\partial u}{\partial y}\right), \quad \mathrm{T}=1, \quad \mathrm{C}=1 \quad \text { at } \quad \mathrm{y}=0 \\
& u \rightarrow 0, \quad T \rightarrow 0, \quad C \rightarrow 0 \quad \text { at } \quad y \rightarrow \infty
\end{aligned}
$$


where $h=\frac{L_{1} v_{0}^{1^{2}}}{v}$.

\section{Solution Of The Problem:}

To solve the partial differential equations (10), (11) and (12), we reduce them into ordinary differential equations. To obtain the solution we follow the procedure given by Gersten and Gross [14]. Therefore the expressions for velocity, temperature and concentration are assumed in the following form.

$$
\begin{aligned}
& u(y, t)=u_{0}(y)+\varepsilon u_{1}(y) e^{-n t} \\
& T(y, t)=T_{0}(y)+\varepsilon T_{1}(y) e^{-n t} \\
& C(y, t)=C_{0}(y)+\varepsilon C_{1}(y) e^{-n t}
\end{aligned}
$$

Substituting above expressions (14), (15), (16) in the equatins (10), (11), (12) and equating the coefficient of $\varepsilon^{0}$ ,$\varepsilon^{1}$ (neglecting $\varepsilon^{2}$ terms etc.), we obtain the following set of ordinary differential equations.

$u_{0}^{11}(y)+u_{0}^{1}(y)-M_{1} u_{0}(y)=-G_{r} T_{0}(y)-G_{m} C_{0}$

$u_{1}^{11}(y)+u_{1}^{1}(y)-M_{2} u_{1}(y)=-\frac{1}{k_{0}} u_{0}(y)-u_{0}^{1}(y)-G_{r} T_{1}(y)-G_{m} C_{1}(y)$

$T_{0}^{11}(y)+(1+H) P_{r} T_{0}^{1}(y)-R P_{r} T_{0}(y)=0$

$T_{1}^{11}(y)+(1+H) P_{r} T_{1}^{1}(y)-\left(R-\frac{n}{4}\right) P_{r} T_{1}(y)=-P_{r} T_{0}^{1}(y)$

$C_{0}^{11}(y)+S_{c} C_{0}^{1}(y)-K_{c} S_{c} C_{0}(y)=0$

$C_{1}^{11}(y)+S_{c} C_{1}^{1}(y)-\left(K_{c}-\frac{n}{4}\right) S_{c} C_{1}(y)=-S_{c} C_{0}^{1}(y)$

Where

$$
M_{1}=M+\frac{1}{K_{0}} \quad \text { and } \quad M_{2}=M+\frac{1}{K_{0}}-\frac{n}{4}
$$

And the boundary conditions (13) reduce to,

$$
\begin{array}{ll}
u_{0}=h u_{0}^{1}, u_{1}=h u_{1}^{1}, T_{0}=1, T_{1}=0, C_{0}=1, C_{1}=0 & \text { at } y=0 \\
u_{0} \rightarrow 0, u_{1} \rightarrow 0, T_{0} \rightarrow 0, T_{1} \rightarrow 0, C_{0} \rightarrow 0, C_{1} \rightarrow 0 & \text { as } y \rightarrow \infty
\end{array}
$$

The equations from (17) to (22) are second order linear differential equations with constant coefficients. The solutions of these paired equations under the corresponding boundary conditions (23) are,

$$
\begin{aligned}
& C_{0}(y)=e^{-m_{1} y} \\
& C_{1}(y)=A_{1}\left(e^{-m_{1} y}-e^{-m_{2} y}\right) \\
& T_{0}(y)=e^{-m_{3} y} \\
& T_{1}(y)=A_{2}\left(e^{-m_{3} y}-e^{-m_{4} y}\right) \\
& u_{0}(y)=A_{5} e^{-m_{5} y}+A_{3} e^{-m_{3} y}+A_{4} e^{-m_{1} y} \\
& u_{1}(y)=A_{11} e^{-m_{6} y}+A_{6} e^{-m_{5} y}+A_{7} e^{-m_{3} y}+A_{8} e^{-m_{1} y}+A_{9} e^{-m_{4} y}+A_{10} e^{-m_{2} y}
\end{aligned}
$$

Where the constants are given in the appendix;

Introducing (28) and (29) in (14), the velocity field $\mathrm{u}(\mathrm{y}, \mathrm{t})$ is,

$$
\begin{aligned}
& u(y, t)=\left(A_{5} e^{-m_{5} y}+A_{3} e^{-m_{3} y}+A_{4} e^{-m_{1} y}\right)+\varepsilon\left(A_{11} e^{-m_{6} y}+A_{6} e^{-m_{5} y}\right. \\
& \left.+A_{7} e^{-m_{3} y}+A_{8} e^{-m_{1} y}+A_{9} e^{-m_{4} y}+A_{10} e^{-m_{2} y}\right) e^{-n t}
\end{aligned}
$$

Introducing (26) and (27) in (15), the temperature field $\mathrm{T}(\mathrm{y}, \mathrm{t})$ is,

$T(y, t)=\left(e^{-m_{3} y}\right)+\varepsilon A_{2}\left(e^{-m_{3} y}-e^{-m_{4} y}\right) e^{-n t}$ 
Also introducing (24) and (25) in (16), the concentration field $\mathrm{C}(\mathrm{y}, \mathrm{t})$ is,

$$
C(y, t)=\left(e^{-m_{1} y}\right)+\varepsilon A_{1}\left(e^{-m_{1} y}-e^{-m_{2} y}\right) e^{-n t}
$$

SKIN FRICTION:

The expression for the skin-friction $(\tau)$ at the plate is,

$$
\begin{aligned}
\tau=\left(\frac{d u}{d y}\right)_{y=0} & =\left(\frac{d u_{0}}{d y}\right)_{y=0}+\varepsilon\left(\frac{d u_{1}}{d y}\right)_{y=0} e^{-n t} \\
& =A_{12}+\varepsilon A_{13} e^{-n t}
\end{aligned}
$$

RATE OF HEAT TRANSFER:

The expression for the rate of heat transfer in terms of Nusselt number $\left(\mathrm{N}_{\mathrm{u}}\right)$ is,

$$
\begin{aligned}
N_{u}=\left(\frac{d T}{d y}\right)_{y=0} & =\left(\frac{d T_{0}}{d y}\right)_{y=0}+\varepsilon\left(\frac{d T_{1}}{d y}\right)_{y=0} e^{-n t} \\
& =-m_{3}+\varepsilon A_{14} e^{-n t}
\end{aligned}
$$

\section{RATE OF MASS TRANSFER:}

The expression for the rate of heat transfer in terms of Sherwood number ( $\mathrm{Sh}$ ) is

$$
\begin{aligned}
S_{h}=\left(\frac{d C}{d y}\right)_{y=0} & =\left(\frac{d C_{0}}{d y}\right)_{y=0}+\varepsilon\left(\frac{d C_{1}}{d y}\right)_{y=0} e^{-n t} \\
& =-m_{1}+\varepsilon A_{15} e^{-n t}
\end{aligned}
$$

\section{Results And Discussions:}

To assess the physical depth of the problem, the effects of various parameters like slip parameter $\mathrm{h}$, Grashof number Gr, Magnetic parameter M, Permeability of Porous medium Ko, Heat source parameter H, Radiation parameter R, Chemical reaction parameter Kc, Modified Grashof number Gm on Velocity distribution, Temperature distribution and Concentration distribution are studied in figures 1-12, while keeping the other parameters as constants. The variations in skin friction, the rate of heat transfer in the form of Nusselt number and the rate of mass transfer in the form of Sherwood number are studied through the tables 1-3. Figure 1 depicts the velocity profiles with the variations in slip parameter $h$, it is observed that the significance of the velocity is high near the plate and there after it decreases and reaches to the stationery position at the other side of the plate. As expected velocity increases with an increase in slip parameter $h$. The effects of Grashof number Gr on velocity distribution are presented in figure 2.

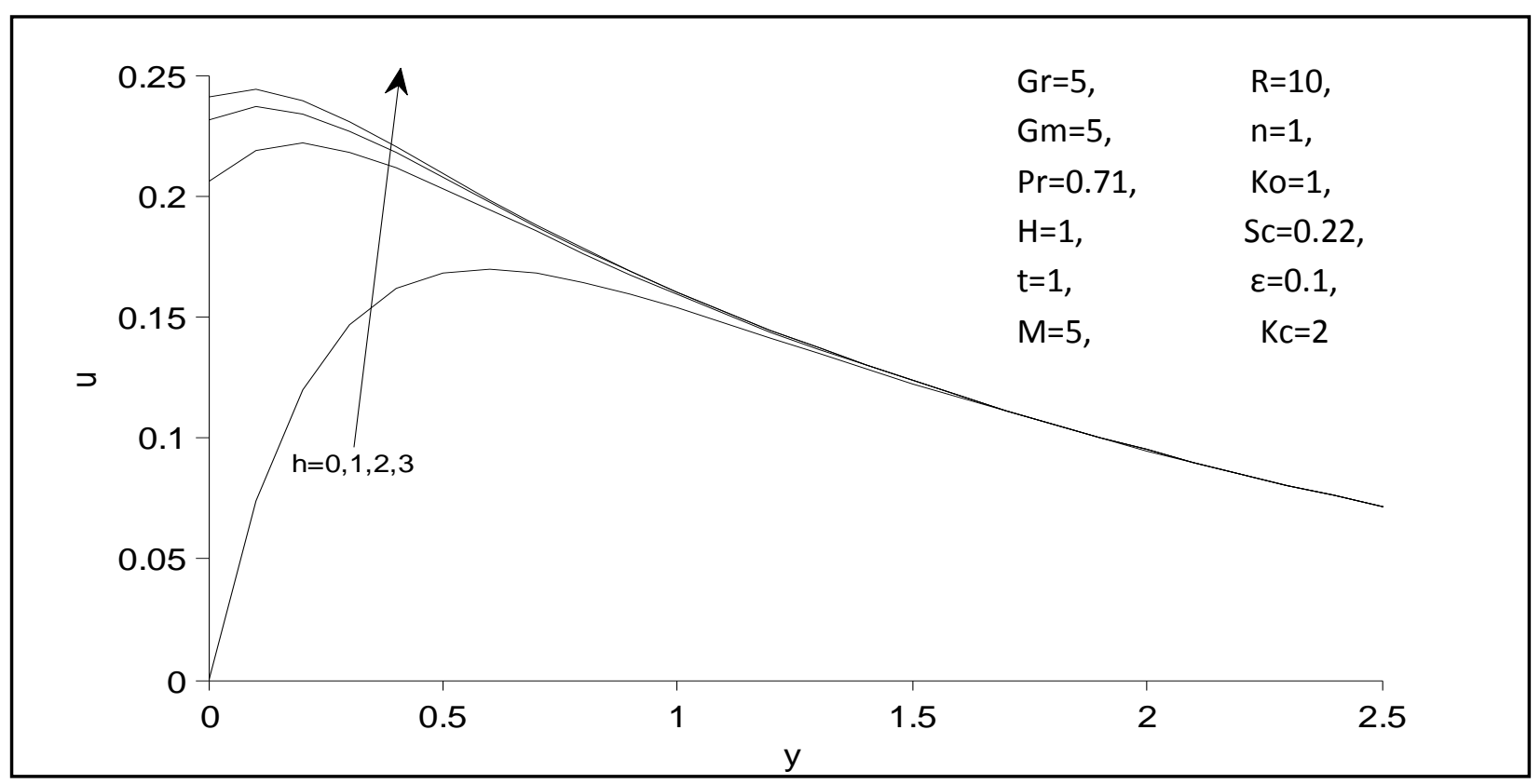

Fig.1.Effects of slip parameter $h$ on velocity 


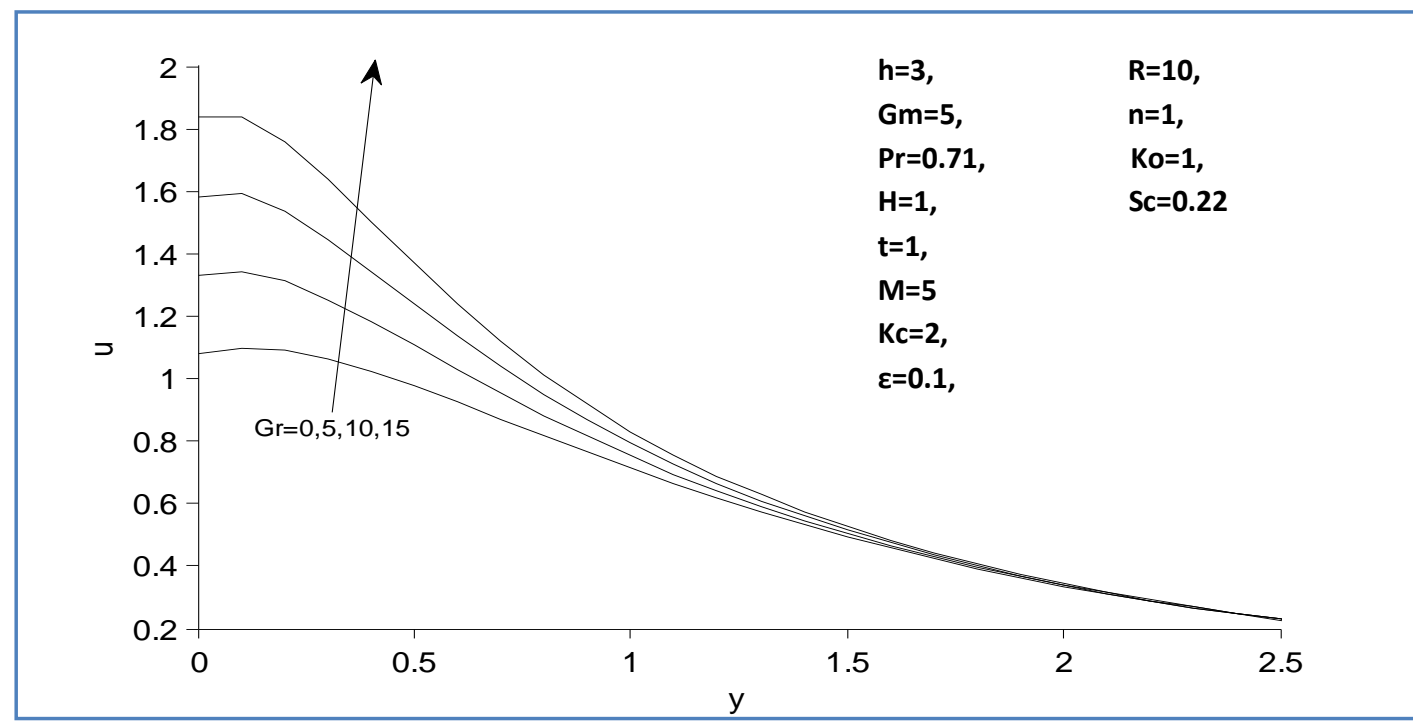

Fig.2.Effects of Grashof number Gr on velocity

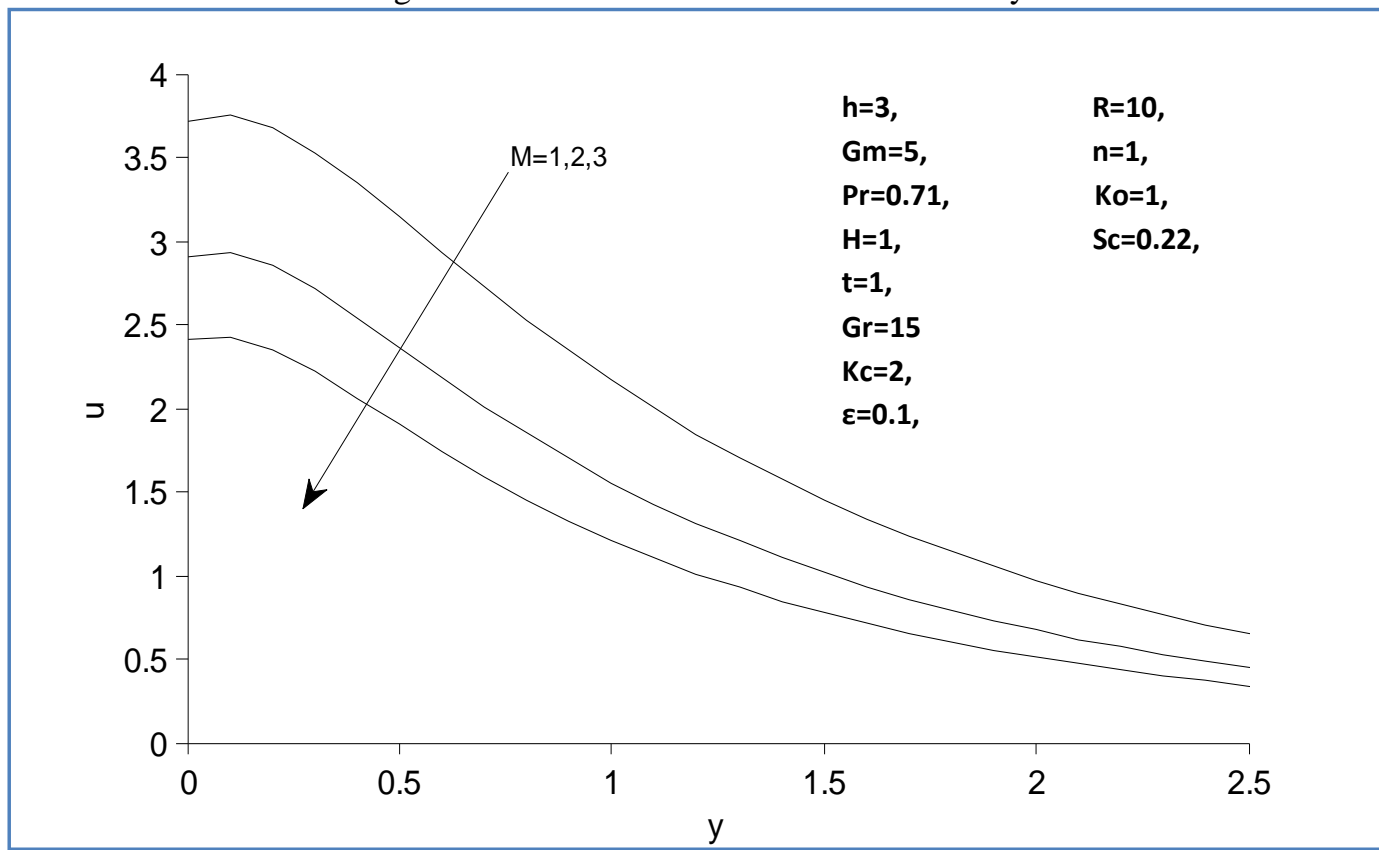

Fig.3.Effects of Magnetic parmeter M on velocity

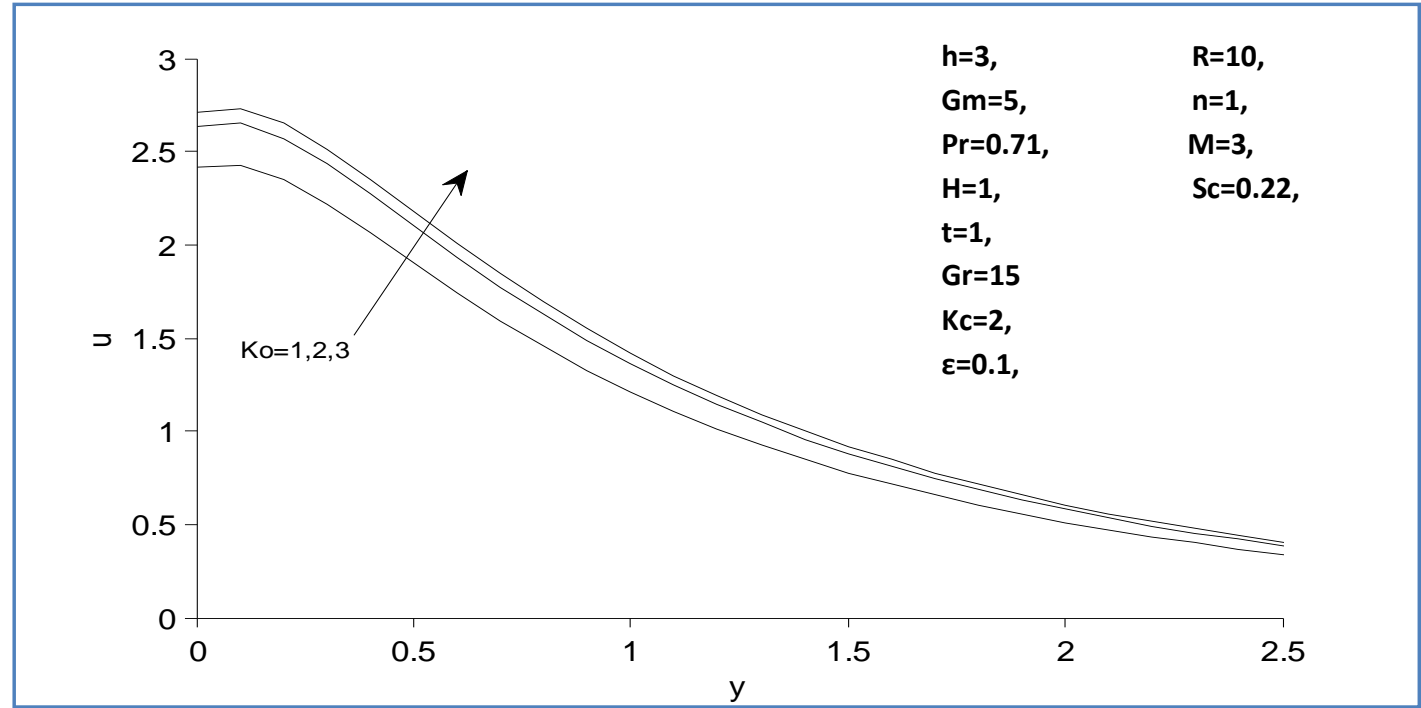

Fig.4.Effects of Permeability of Porus medium Ko on velocity 


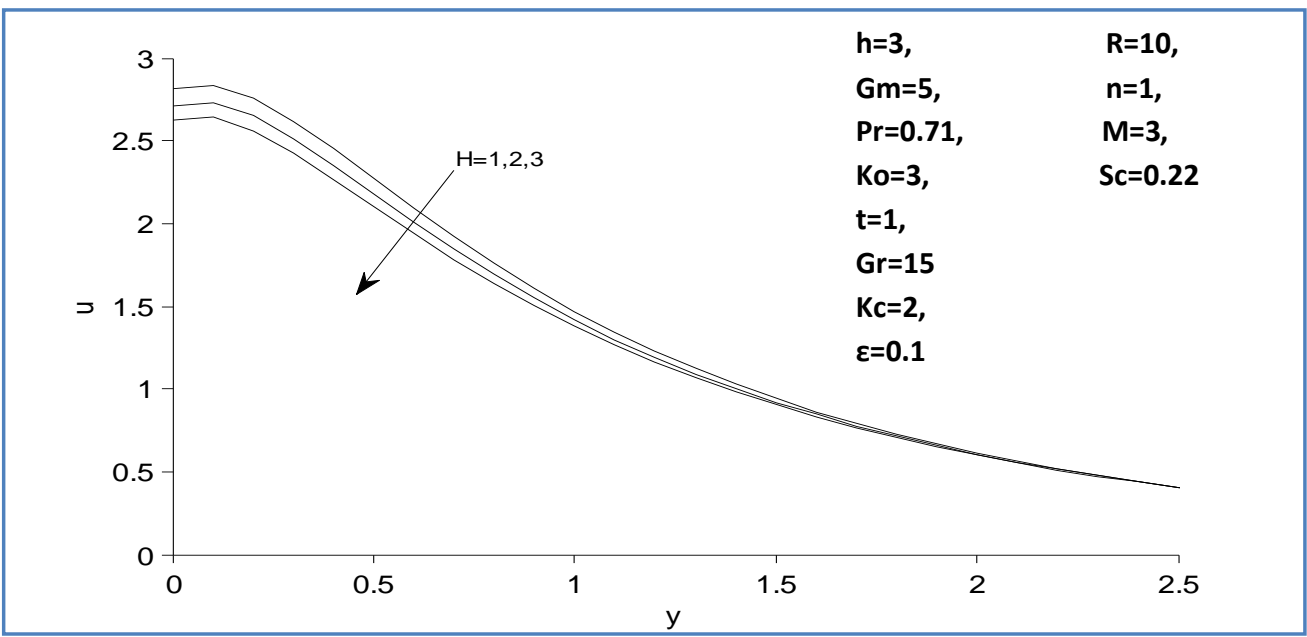

Fig.5.Effects of Heat source parameter $\mathrm{H}$ on velocity

From this figure it is noticed velocity increases as an increase in Gr. In figure 3 the effects of magnetic parameter $\mathrm{M}$ on velocity is shown. From this figure it is noticed that velocity decreases as $\mathrm{M}$ increases. Since, the applied magnetic field acts as Lorentz's force which drags the velocity. In figure 4 the velocity increases as Permeability of Porous medium Ko increases. In figure 5 the velocity decreases as Heat source parameter $\mathrm{H}$ increases. From figure 6 it is observed that the velocity decreases as Radiation parameter R increases. In figure 7 the velocity decreases as chemical reaction parameter $\mathrm{Kc}$ increases. In figure 8 the velocity increases as Modified Grashof number $\mathrm{Gm}$ increases. In the figures 9, 10 the Temperature distribution decreases as Radiation parameter R and Heat source parameter H increase respectively. Similarly, in the figures 11, 12 the Concentration decreases as Schmidt number Sc andchemical reaction parameter Kc increase respectively. To be realistic, the numerical values of Prandtl number $\operatorname{Pr}$ are chosen as $\operatorname{Pr}=0.71$, and $\operatorname{Pr}=7.00$, which correspond to air and water at $20^{\circ} \mathrm{C}$ respectively. The numerical values of the remaining parameters are chosen arbitrarily.

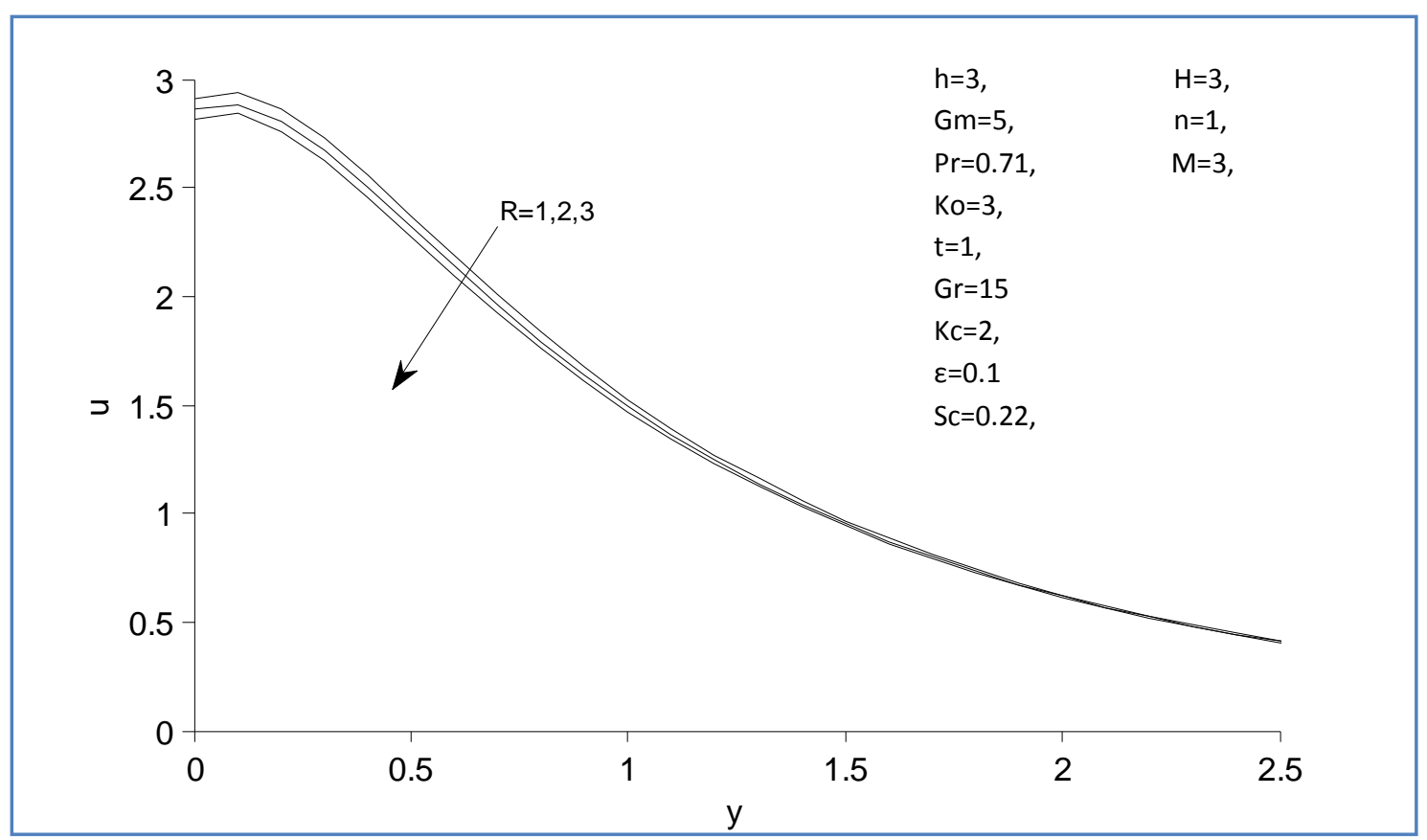

Fig.6.Effectsof Radiation parameter $\mathrm{R}$ on velocity 


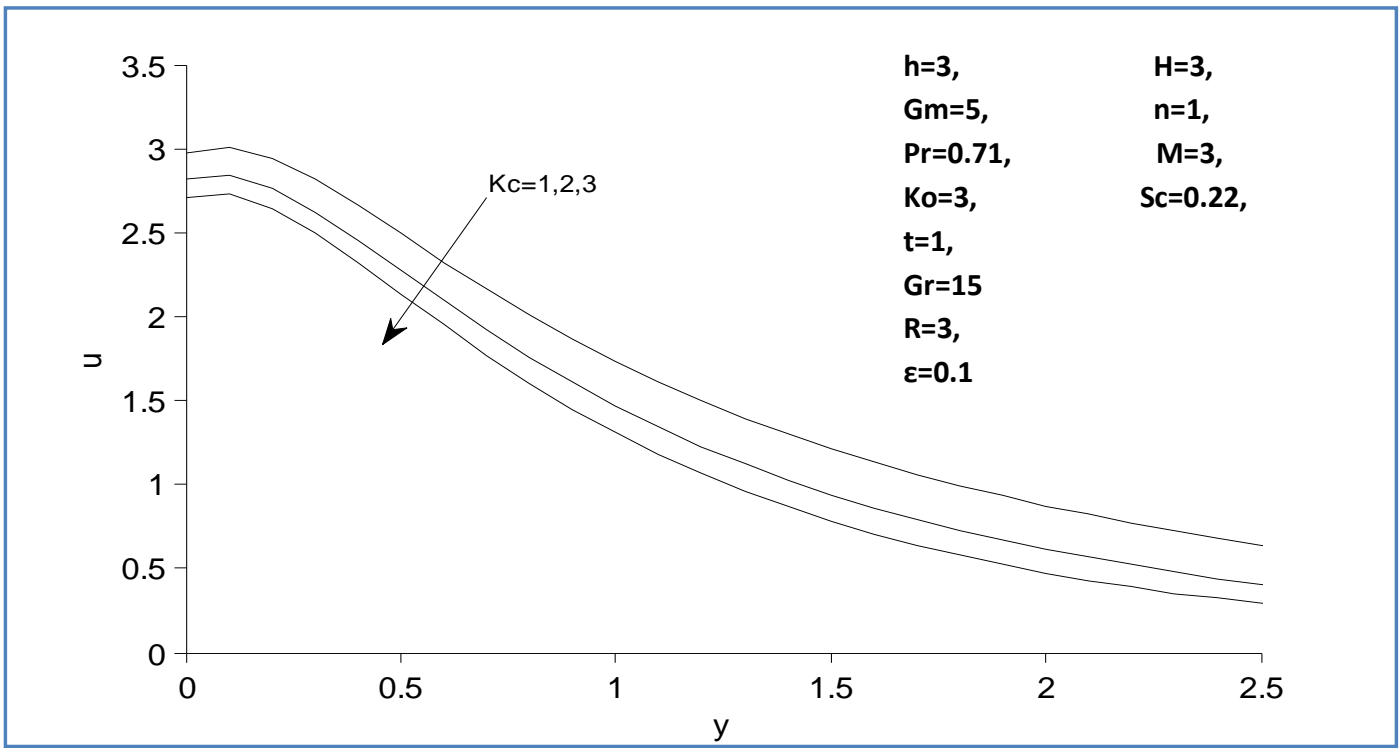

Fig.7.Effectsof Chemical reaction parameter Kc on velocity

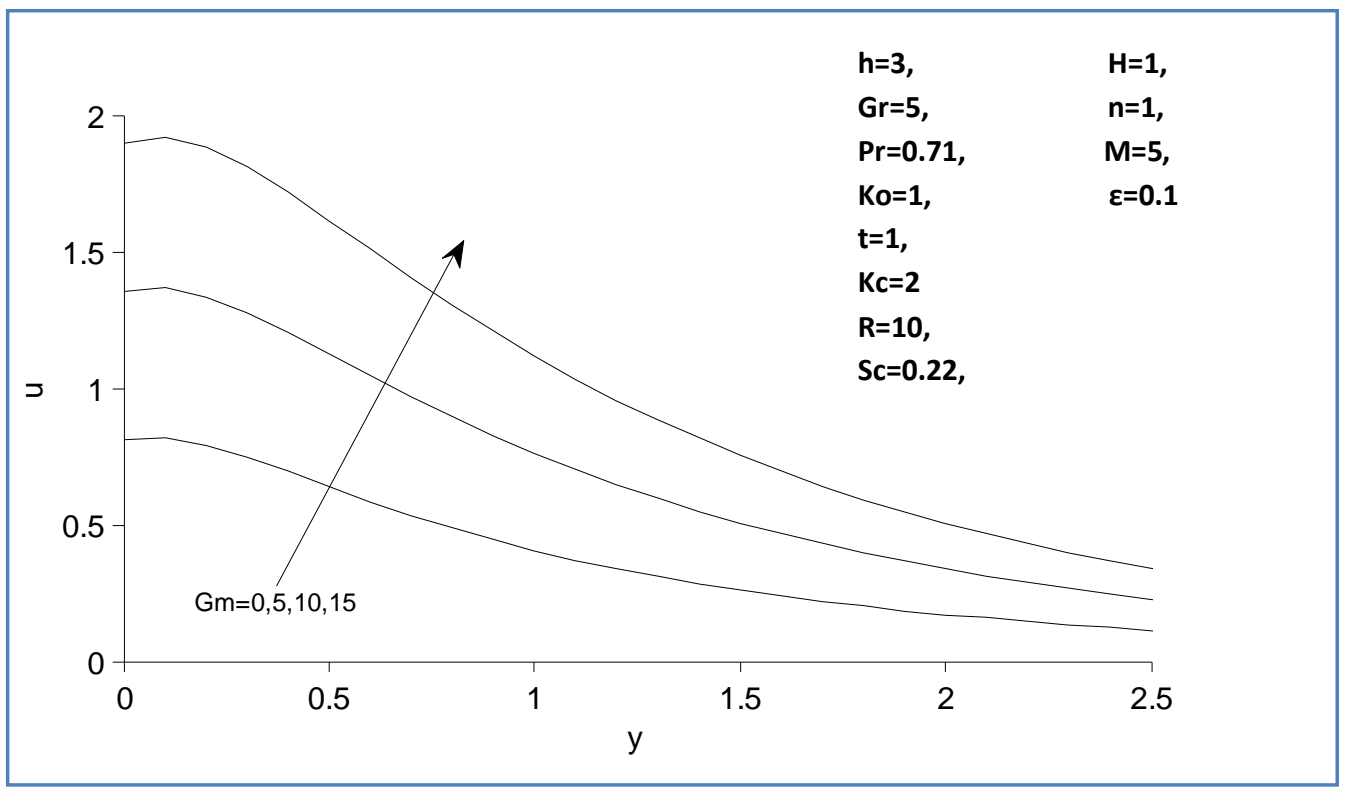

Fig.8.Effectsof modified Grashof number Gm on velocity

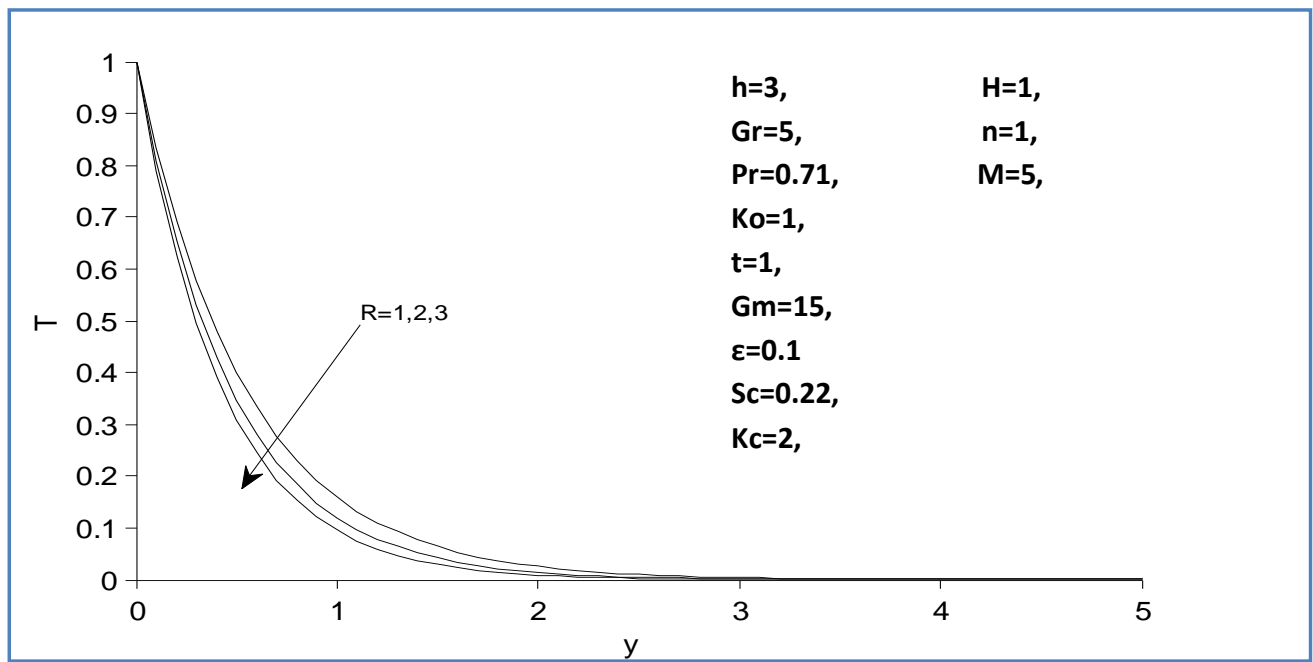

Fig.9.Effectsof Radiation parameter R on Temperature 


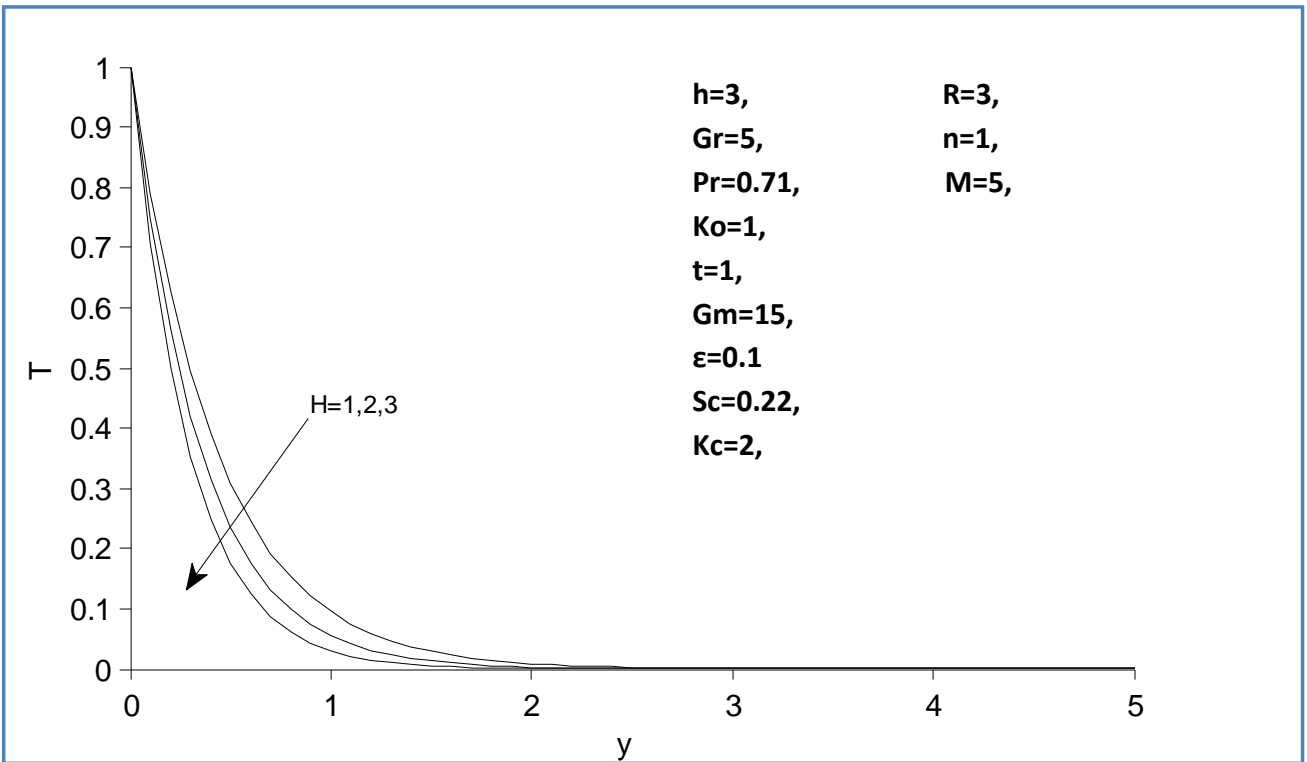

Fig.10.Effects of Heat source parameter $\mathrm{H}$ on Temperature

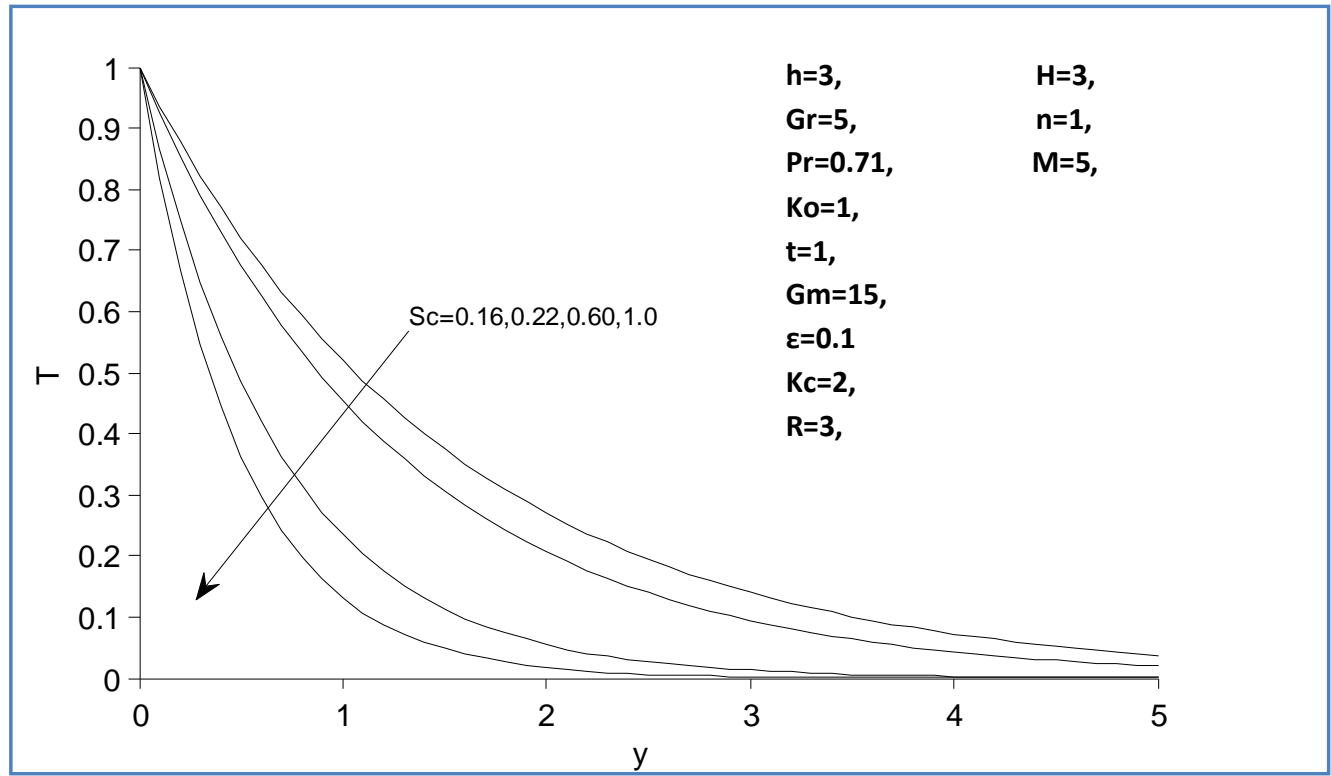

Fig.11.Effects of Schimidt number Sc on Concentration

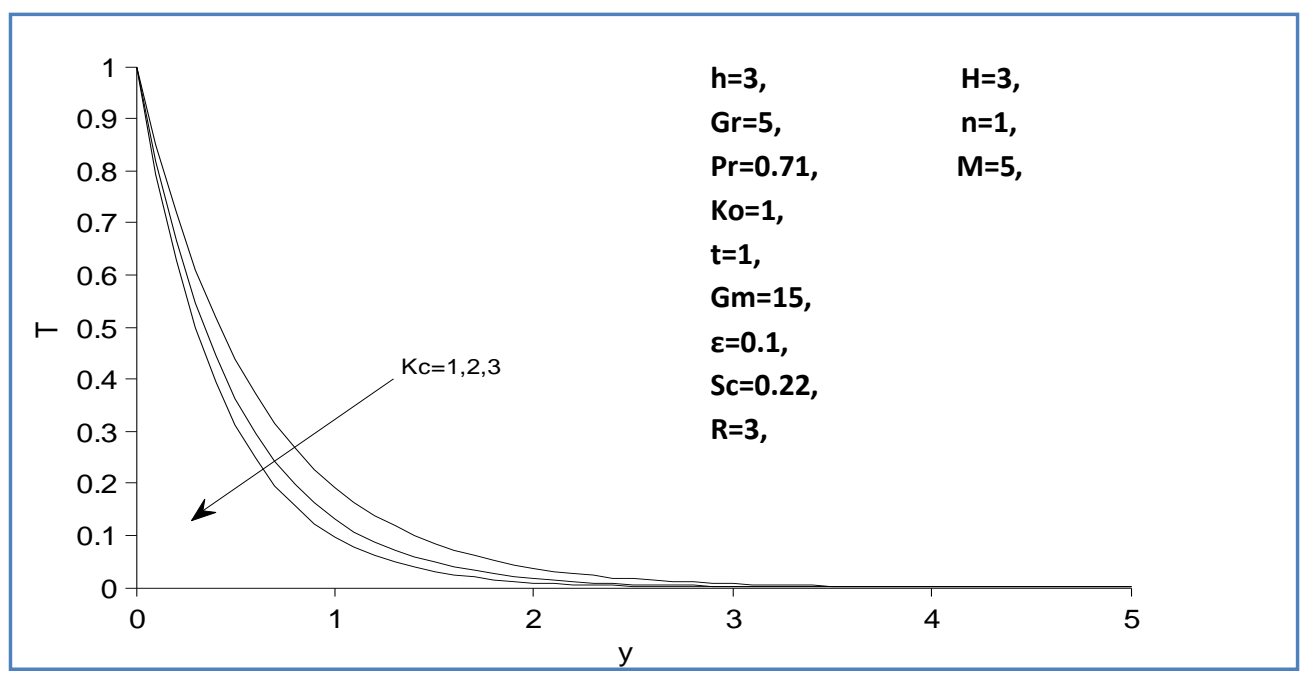


Fig.12.Effects of Chemical reaction parameter Kc on Concenterature

\begin{tabular}{|l|l|l|l|l|l|l|l|l|l|}
\hline $\mathrm{M}$ & Ko & $\mathrm{H}$ & $\mathrm{h}$ & $\mathrm{R}$ & $\mathrm{Gr}$ & $\mathrm{Gm}$ & $\mathrm{Kc}$ & $\mathrm{Sc}$ & $\boldsymbol{\tau}$ \\
\hline 0.5 & 20 & 0.1 & 0.1 & 1 & 10 & 10 & 0.5 & 0.22 & $\mathbf{1 5 . 3 0 1 3}$ \\
\hline 1.0 & 20 & 0.1 & 0.1 & 1 & 10 & 10 & 0.5 & 0.22 & $\mathbf{1 2 . 1 6 7 8}$ \\
\hline 2.0 & 20 & 0.1 & 0.1 & 1 & 10 & 10 & 0.5 & 0.22 & $\mathbf{9 . 1 9 4 6}$ \\
\hline 0.5 & 50 & 0.1 & 0.1 & 1 & 10 & 10 & 0.5 & 0.22 & $\mathbf{1 5 . 5 5 4 2}$ \\
\hline 0.5 & 80 & 0.1 & 0.1 & 1 & 10 & 10 & 0.5 & 0.22 & $\mathbf{1 5 . 6 1 9 3}$ \\
\hline 0.5 & 20 & 0.5 & 0.1 & 1 & 10 & 10 & 0.5 & 0.22 & $\mathbf{1 4 . 7 5 2 6}$ \\
\hline 0.5 & 20 & 1.0 & 0.1 & 1 & 10 & 10 & 0.5 & 0.22 & $\mathbf{1 4 . 1 8 0 8}$ \\
\hline 0.5 & 20 & 0.1 & 0.5 & 1 & 10 & 10 & 0.5 & 0.22 & $\mathbf{1 0 . 1 6 7 8}$ \\
\hline 0.5 & 20 & 0.1 & 1.0 & 1 & 10 & 10 & 0.5 & 0.22 & $\mathbf{7 . 1 6 1 8}$ \\
\hline 0.5 & 20 & 0.1 & 1.0 & 2 & 10 & 10 & 0.5 & 0.22 & $\mathbf{4 . 4 9 9 9}$ \\
\hline 0.5 & 20 & 0.1 & 1.0 & 3 & 10 & 10 & 0.5 & 0.22 & $\mathbf{3 . 2 8 0 4}$ \\
\hline 0.5 & 20 & 0.1 & 1.0 & 1 & 20 & 10 & 0.5 & 0.22 & $\mathbf{9 . 5 1 7 4}$ \\
\hline 0.5 & 20 & 0.1 & 1.0 & 1 & 30 & 10 & 0.5 & 0.22 & $\mathbf{1 1 . 8 7 3 0}$ \\
\hline 0.5 & 20 & 0.1 & 1.0 & 1 & 10 & 20 & 0.5 & 0.22 & $\mathbf{1 1 . 9 6 8 1}$ \\
\hline 0.5 & 20 & 0.1 & 1.0 & 1 & 10 & 30 & 0.5 & 0.22 & $\mathbf{1 6 . 7 7 4 4}$ \\
\hline 0.5 & 20 & 0.1 & 1.0 & 1 & 10 & 10 & 1.0 & 0.22 & $\mathbf{6 . 5 2 1 4}$ \\
\hline 0.5 & 20 & 0.1 & 1.0 & 1 & 10 & 10 & 1.5 & 0.22 & $\mathbf{6 . 1 2 7 5}$ \\
\hline 0.5 & 20 & 0.1 & 1.0 & 1 & 10 & 10 & 1.5 & 0.62 & $\mathbf{4 . 7 1 6 8}$ \\
\hline
\end{tabular}

Table 1: Skin friction at the plate when $\operatorname{Pr}=0,71, \mathrm{n}=0.5, \mathrm{t}=1$ and $\varepsilon=0.1$

\begin{tabular}{|l|l|l|l|}
\hline $\operatorname{Pr}$ & H & R & Nu \\
\hline 0.71 & 0.1 & 1.0 & $\mathbf{- 1 . 3 5 0 6}$ \\
\hline 7.00 & 0.1 & 1.0 & $\mathbf{- 8 . 9 1 2 7}$ \\
\hline 0.71 & 0.5 & 1.0 & $\mathbf{- 1 . 5 6 3 1}$ \\
\hline 0.71 & 0.1 & 2.0 & $\mathbf{- 1 . 3 5 0 6}$ \\
\hline
\end{tabular}

Table 2. Rate of heat transfer at the plate

\begin{tabular}{|l|l|l|}
\hline Kc & Sc & Sh \\
\hline 0.2 & 0.22 & $\mathbf{- 0 . 3 5 8 3}$ \\
\hline 0.2 & 0.62 & $\mathbf{- 0 . 8 1 3 7}$ \\
\hline 0.4 & 0.22 & $\mathbf{- 0 . 4 3 6 1}$ \\
\hline 0.6 & 0.22 & $\mathbf{- 0 . 4 9 8 7}$ \\
\hline
\end{tabular}

Table 3. Rate of mass transfer at the plate

\section{Conclusions:}

1. The Velocity increases with an increase in slip parameter $h$, Grashof number $G_{r}$, modified Grashof number $G_{m}$, Permeability of Porous medium Ko and it shows the reverse effect with an increase in magneticparameter M, Heat source parameter $\mathrm{H}$, Radiation parameter $\mathrm{R}$, Chemical reaction parameter Kc.

2. The Temperature decreases with an increase in Radiation parameter $\mathrm{R}$ and Heat source parameter $\mathrm{H}$.

3. The Concentration decreases with an increase in Schmidt number Sc and Chemical reaction parameter Kc.

\section{References:}

[1]. Chamaka A.J, Hydro magnetic combined heat and mass transfer by natural convection from a permeable surface embedded in a fluid saturated porous medium, ,Int. J. Num.Methods for heat and fluid flow, 10(5),pp. 455-476, 2000.

[2]. Chamkha A.J, Khaled A.R., Hydromagnetic combined heat and mass transfer by natural convection from a permeable surface embedded in fluid saturated porous medium", International Journal of Numerical methods for Heat and Fluid Flow, 10(5), pp. 455476, 2000.

[3]. Chamkha A.J., MHD flow of a uniformly stretched vertical permeable surface in the presence of heat generation/absorption and a chemical reaction, Int.Commun. Heat mass transfer, 30, (No.3), 413-422, 2003.

[4]. Ede A.J., Advances in Heat Transfer, Academic Press, New York (1967).

[5]. Gupta M and Sharma S., "MHD flow of viscous fluid through a porous medium bounded by an oscillating porous plate in slip flow regime", Acta Ciencia Indica, 17M, 389-394(1991).

[6]. Hossain A.M, Alim M.A. and Rees, D.A.S.,(1999). Effect of radiation on free convection from a porous a vertical plate, International journal of Heat and Mass Transfer, 42: 181-191, 1999.

[7]. Ibrahim, F. S., Elaiw, A. M., and Bakr, A. A. Effect of the chemical reaction and radiation absorption on the unsteady MHD free convection flow past a semi infinite vertical permeable moving plate with heat source and suction: Comm. Nonlinear Sci. Numer. Simul13 (6), 1056-1066 (2008)

[8]. Jaiswal. B.S and Soundalgekar. V.M., Transient convective flow past an infinite vertical oscillating plate with temperature gradient dependent heat source: Bul. All India Math. Soc., 15, 25-29 (2000). 
[9]. Kandasamy, R., Periasamy, K., and Prabhu, K. K. S. Chemical reaction, heat and mass transfer on MHD flow over a vertical stretching surface with heat source and thermal stratification effects: Int. J. Heat Mass Transfer 48, 4557-4561 (2005).

[10]. Kim. J Youn, Unsteady MHD convective heat transfer past a semi-infinite vertical porous moving plate with variable suction, International Journal of Engineering Sciences 38, pp. 833-845, 2000.

[11]. Kumar. K., Varshney. M. and Varshney.C.L., Elastico-viscous stratified flucturating Hartmann flow through a porous medium past an infinteregid plane in slip regime: JMACT, 20, 65-71 (1987).

[12]. Muthucumarswamy R. and Kumar G.S.,(2004). Heat and Mass Transfer effects on moving vertical plate in the presence of thermal radiation, Theoret. Appl. Mach., 31(1), 35-46, 2004.

[13]. Pal, D. and Talukda, B. (2009). Perturbation analysis of unsteady Magneto hydrodynamic convective heat and mass transfer in a boundary layer slip flow past a vertical permeable plate with thermal radiation and chemical reaction: Communications in Nonlinear Science and Numerical Simulation, In Press, Corrected Proof, Available online 17 July 2009

[14]. Postelnicu, A. Influence of chemical reaction on heat and mass transfer by natural convection from vertical surfaces in porous media considering Soret and Dufour effects: Heat and Mass Transfer 43, , 595-602 (2007).

[15]. Raju M.C, Varma S.V.K, Reddy N.A., "MHD Thermal diffusion Natural convection flow between heated inclined plates in porous medium", 2011, Journal on Future Engineering and Technology.Vol.6, No.2, pp.45-48.

[16]. Raju M.C, Varma S.V.K, Reddy N.A., "Radiation and mass transfer effects on a free convection flow through a porous medium bounded by a vertical surface", Journal of Future Engineering and Technology, Vol. 7, No: 2, pp. 7-12 2012. ISSN: 0975-7783.

[17]. Raju M.C, Varma S.V.K, Reddy P.V and Sumon Saha, " Soret effects due to Natural convection between Heated Inclined Plates with Magnetic Field", Journal of Mechanical Engineering, Vol. ME39,No.Dec 2008,43-48.

[18]. Sharma P.R, Kumar N. and Sharma P., (2011).Influence of chemical reaction and radiation on unsteady MHD free convection flow and mass transfer through viscous incompressible fluid past a heated vertical plate immersed in porous medium in the presence of heat source, Appl. Math. Sciences,5, (No.46), 2249-2260, 2011.

[19]. Sharma, P. K. (2005). Fluctuating thermal and mass diffusion on unsteady free convective flow past a vertical plate in slip-flow regime:Latin American Applied Research 35, 313-319

[20]. Sharma, P. K. and Chaudhary, R. C. (2003). Effect of variable suction on transient free convection viscous incompressible flow past a vertical plate with periodic temperature variations in slip-flow regime: Emirates J. Engineering Research, 8, 33-38.

[21]. Singh A.K. and Kumar N., Free convection flow past an exponentially accelerated vertical plate, Astrophysics and Space science , 98, 245-258, 2000.

[22]. Singh. Atul Kumar, Singh. N.P and Singh. R.V, MHD Flow of a dusty viscoelastic liquid (rivlin-Ericksen) through a porous medium bounded by an oscillating porous plate in slip flow regime: The Math. Edu., 34, 53-55(2000).

[23]. Singh. N.P, Singh.R.V and Singh. Atul Kumar, Flow of a viscoelastic fluid through a porous medium near an oscillating plate in slip flow regime in the presence of electromagnetic field : Ind.J. Theo. Phys., 47, 203-209(1999).

[24]. Singh. N.P, Singh.R.V and Singh. Atul Kumar, Flow of a dusty viscoelastic fluid through a porous medium near an oscillating porous plate in slip flow regime: JMACT, 31, 99-108(1998).

[25]. Srinivas. S \& Muthuraj.R., MHD flow with slip effects and temperature-dependent heat source in a vertical wavy porous space, chem. eng. comm., 197,1387-1403, 2010.

[26]. Sudhakar reddy T, Raju M.C \&.Varma S.V.K, “ The effect of slip condition, Radiation and chemical reaction on unsteady MHD periodic flow of a viscous fluid through saturated porous medium in a planar channel, Journal on Mathematics, Vol.1, No.1, 2012,

[27]. Sudhakar reddy T, Raju M.C \&.Varma S.V.K, "Unsteady MHD free convection Oscillatory couette flow through a porous medium with periodic wall temperature in presence of chemical reaction and thermal radiation", International Journal of Science and advances technologies, Vol.1, no.10, pp. 51-58, 2011

[28]. Taneja. R and Jain. N.C., Effects of magnetic field on free convection and mass transfer flow through porous medium and variable permeability in slip flow regime: Jnanabh, 31/32, 69-81 (2002).

[29]. Yamamoto. K and Iwamura. N., Flow with convective acceleration through a porous medium: J. Engg.Math., 10, 41-54(1976).

[30]. Yamamoto.K and Yoshidha.Z., Flow through a porous wall with convective acceleration: J.Phys. Soc., Japan, 37, 774-786(1974).

\section{APPENDIX:}

$$
\begin{aligned}
& m_{1}=\frac{S_{c}+\sqrt{S_{c}{ }^{2}+4 K_{c} S_{c}}}{2}, m_{2}=\frac{S_{c}+\sqrt{S_{c}{ }^{2}+S_{c}\left(4 K_{c}-n\right)}}{2}, m_{3}=\frac{(1+H) P_{r}+\sqrt{(1+H)^{2} P_{r}^{2}+4 R P_{r}}}{2} \\
& m_{4}=\frac{(1+H) P_{r}+\sqrt{(1+H)^{2} P_{r}^{2}+(4 R-n) P_{r}}}{2}, m_{5}=\frac{1+\sqrt{1+4 M_{1}}}{2}, m_{6}=\frac{1+\sqrt{1+4 M_{2}}}{2} \text {, } \\
& A_{1}=\frac{S_{c} m_{1}}{m_{1}^{2}-S_{c} m_{1}-\left(K_{c}-\frac{n}{4}\right) S_{c}}, A_{2}=\frac{P_{r} m_{3}}{m_{3}^{2}-(1+H) P_{r} m_{3}-\left(R-\frac{n}{4}\right) P_{r}}, A_{3}=-\frac{G_{r}}{m_{3}{ }^{2}-m_{3}-M_{1}} \\
& A_{4}=-\frac{G_{m}}{m_{1}^{2}-m_{1}-M_{1}}, A_{5}=-\left(\frac{A_{3}\left(1+h m_{3}\right)+A_{4}\left(1+h m_{1}\right)}{1+h m_{5}}\right), A_{6}=\frac{\left(A_{5} m_{5}-\frac{A_{5}}{K_{0}}\right)}{m_{5}{ }^{2}-m_{5}-M_{2}} \\
& A_{7}=\frac{\left(A_{3} m_{3}-A_{2} G_{r}-\frac{A_{3}}{K_{0}}\right)}{m_{3}^{2}-m_{3}-M_{2}}, A_{8}=\frac{\left(A_{4} m_{1}-A_{1} G_{m}-\frac{A_{4}}{K_{0}}\right)}{m_{1}{ }^{2}-m_{1}-M_{2}}, A_{9}=\frac{A_{2} G_{r}}{m_{4}{ }^{2}-m_{4}-M_{2}}, A_{10}=\frac{A_{1} G_{m}}{m_{2}{ }^{2}-m_{2}-M_{2}} \\
& A_{11}=-\left(\frac{A_{6}\left(1+h m_{5}\right)+A_{7}\left(1+h m_{3}\right)+A_{8}\left(1+h m_{1}\right)+A_{9}\left(1+h m_{4}\right)+A_{10}\left(1+h m_{2}\right)}{1+h m_{6}}\right) \\
& A_{12}=-A_{5} m_{5}-A_{3} m_{3}-A_{4} m_{1}, A_{13}=-A_{11} m_{6}-A_{6} m_{5}-A_{7} m_{3}-A_{8} m_{1}-A_{9} m_{4}-A_{10} m_{2} \\
& A_{14}=A_{2} m_{4}-A_{2} m_{3}, A_{15}=A_{1} m_{2}-A_{1} m_{1}
\end{aligned}
$$

